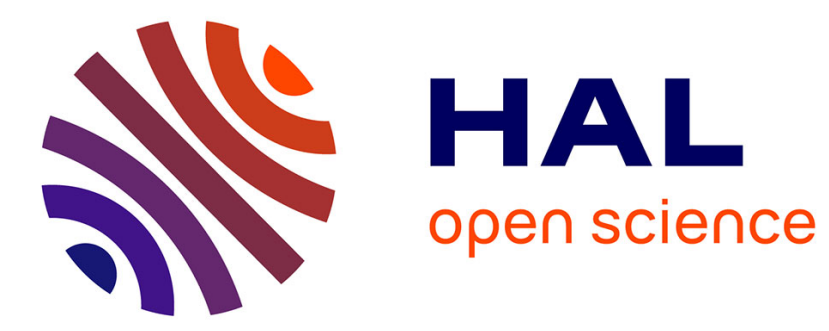

\title{
Size effect in Quincke rotation: a numerical study
}

Francois Peters, Laurent Lobry, A. Khayari, Elisabeth Lemaire

\section{To cite this version:}

Francois Peters, Laurent Lobry, A. Khayari, Elisabeth Lemaire. Size effect in Quincke rotation: a numerical study. Journal of Chemical Physics, 2009, 130 (19), pp.194905.1-194905.8. 10.1063/1.3134046 . hal-00436370

\section{HAL Id: hal-00436370 https://hal.science/hal-00436370}

Submitted on 3 Dec 2009

HAL is a multi-disciplinary open access archive for the deposit and dissemination of scientific research documents, whether they are published or not. The documents may come from teaching and research institutions in France or abroad, or from public or private research centers.
L'archive ouverte pluridisciplinaire HAL, est destinée au dépôt et à la diffusion de documents scientifiques de niveau recherche, publiés ou non, émanant des établissements d'enseignement et de recherche français ou étrangers, des laboratoires publics ou privés. 
Size effect in Quincke rotation: a numerical study.

\author{
F. Peters, L. Lobry, A. Khayari and E. Lemaire \\ LPMC - CNRS Université de Nice \\ 06 10/8 Nice cedex 2 - France
}

\begin{abstract}
This paper deals with the Quincke rotation of small insulating particles. This DC electro-rotation of insulating objects immersed in a slightly conducting liquid is usually explained by looking at the action of the free charges present in the liquid. Under the effect of the DC electric field, the charges accumulate at the surface of the insulating particle which, in turn, acquires a dipole moment in the direction opposite to that of the field and begins to rotate in order to flip its dipole moment. In the classical Quincke model, the charge distribution around the rotor is supposed to be purely superficial. A consequence of this assumption is that the angular velocity does not depend on the rotor size. Nevertheless, this hypothesis holds only if the rotor size is much larger than the characteristic ion layer thickness around the particle. In the opposite case, we show thanks to numerical calculations that the bulk charge distribution has to be accounted for to predict the electro-mechanical behaviour of the rotor. We consider the case of an infinite insulating cylinder whose axis is perpendicular to the DC electric field. We use the finite element method to solve the conservation equations for the positive and the negative ions coupled with Navier-Stokes and Poisson equations. Doing so, we compute the bulk charge distribution and the velocity field in the liquid surrounding the cylinder. For sufficiently small cylinders, we show that the smaller the cylinder is, the smaller its angular velocity when submitted to a DC electric field. This size effect is shown to originate both in ion diffusion and electro-migration in the charge layer. At last, we propose a simple analytical model which allows calculating the angular velocity of the rotor when electro-migration is present but weak and diffusion can be neglected.
\end{abstract}




\section{Introduction}

When a non conducting particle immersed in a low conducting liquid is submitted to a sufficiently high DC field, it can rotate spontaneously around itself along any axis perpendicular to the electric field. This symmetry break is known as Quincke rotation from the name of the man who first observed it at the end of the $19^{\text {th }}$ century [1]. As explained by Secker and Scialom [2] and later by Melcher [3], the mechanism responsible for Quincke rotation deals with the action of the free electric charges which are contained in the liquid. Indeed, when the E field, $\mathbf{E}_{\mathbf{0}}$, is applied, the charges contained both in the liquid and in the particle migrate under coulombic force. Then, as stated by Jones [4], depending on the relative magnitude of the charge relaxation times in the liquid and in the particle, the superficial charge distribution which builds at the particle/liquid interface is equivalent to a dipole, $\mathbf{P}$, which is either in the direction of the field or in the opposite direction. In the following, we will call $\mathbf{P}$ the retarded dipole moment since, as it is associated with the charge distribution at the particle/liquid interface, it evolves with a finite characteristic time, named Maxwell-Wagner time, $\tau_{\mathrm{MW}}$.

The charge relaxation times in the liquid, $\tau_{1}$, and in the particle, $\tau_{2}$, are given by the ratio of the permittivity, $\varepsilon_{i}$, to the conductivity, $\sigma_{i}, i=1,2$ refers to the liquid and the particle respectively. When $\tau_{2}<\tau_{1}$, the induced dipole is along the $\mathrm{E}$ field direction and the configuration is stable. In the opposite case, i.e $. \tau_{2}>\tau_{1}$ (for instance, when an insulating particle is immersed in a conducting liquid), the dipole, $\mathbf{P}$, which has been created by the accumulation of the charges at the particle/liquid interface is opposite to the E field direction (figure 1.a.). In this last case, if the particle is slightly rotated, the deviation of its dipole moment $\mathbf{P}$ produces a torque, $\Gamma^{\mathbf{E}}=\mathbf{P} \times \mathbf{E}_{\mathbf{0}}$, which tends to increase the angular tilt further. So, if 
the E field intensity is high enough for the electric torque to balance the viscous resistant torque exerted by the surrounding liquid on the particle, the particle will rotate continuously around itself with an axis pointing in any direction perpendicular to DC field (figure 1.b.). A noteworthy feature of Quincke rotation is that the angular velocity of the particle is expected not to depend on its size.

Quincke rotation has many implications on mechanical systems. Dielectric motors consisting of a cylindrical rotor placed between two plane electrodes have been proposed $[2,5]$. The behaviour of an ensemble of particles undergoing Quincke rotation is also of interest. These last few years, we have been working on the consequences of Quincke rotation on the electrical and mechanical behaviours of a suspension. In particular, we have demonstrated that the particle rotation can be responsible for a sensitive increase of the conductivity of a suspension [6] since the particle rotation facilitates the ion migration. On the other hand, the particle rotation causes a substantial decrease of the apparent viscosity of a suspension [7]. These two effects have been both demonstrated with suspensions containing particles whose diameter is comprised between 50 and 100 microns. It would be interesting to decrease the particle size since the control of the conductivity or of the viscosity of a suspension may have valuable applications in xerography where the pigments are submicronic [8]. Also, it may be of interest to use a single particle as a micro-motor in microfluidics devices [9].

A priori, following the classical model used to predict the electro-mechanical behaviour of a particle undergoing Quinke rotation, the size of the particle does not play any role. Nevertheless, a common hypothesis made in this model consists in assuming that the electric charge distribution created by the field around the particle is purely superficial. One can wonder if this hypothesis remains valid whatever the particle size. Indeed, because of thermal diffusion, the thickness of the charged layer around the particle is a priori expected to be of 
the order of the Debye length $\sqrt{\mathrm{D} \tau_{1}}$ where $\mathrm{D}$ is the thermal diffusion coefficient of the ions whose typical value in the non polar fluids we study is $2.510^{-11} \mathrm{~m}^{2} \mathrm{~s}^{-1}$ (typically, the viscosity of the fluid is $10 \mathrm{mPa} . \mathrm{s}$. and the ion size 1 nanometer). Since the order of magnitude of the charge relaxation time is comprised between 1 and $100 \mathrm{~ms}$, the charge layer thickness is expected to be in the range of one tenth of a micron up to one micron. As a consequence, one can guess that the rotation of small particles should depend on their size if not much larger than the charge layer thickness. The purpose of the present paper is to study numerically this size effect in Quincke rotation.

While various theoretical and numerical studies deal with the AC electro-rotation of colloidal particles dispersed in an aqueous medium [10], [11], [12], the microscopic analysis of a small particle undergoing Quincke rotation has never been addressed. For the sake of simplicity, we will carry out a 2D numerical study where the rotation of an infinite cylinder whose revolution axis is perpendicular to the applied DC electric field will be considered. Furthermore, only the case of a perfectly insulating cylinder will be considered in the numerical study. In the first part, the usual model of Quincke rotation is reminded. In the second part, we present the electrohydrodynamic equations which have been used to determine the ions distribution around the insulating cylinder and the velocity field in the liquid. The following section is devoted to the presentation of the finite elements method, used to solve the above equations and of the main results concerning the dependence of the cylinder spin rate on its diameter. At last, we propose an analytical extension of the classical Quincke rotation model by accounting for the charge electro-migration inside the ionic layer around the cylinder and we analyse the numerical results thanks to this modified Quincke rotation model. 


\section{Classical treatment of Quincke rotation in two dimensions, macroscopic description.}

We consider an infinite cylinder (radius a, conductivity $\sigma_{2}$, permittivity $\varepsilon_{2}$ ) immersed in a dielectric liquid (viscosity $\eta$, conductivity $\sigma_{1}$, permittivity $\varepsilon_{1}$ ) and subjected to a DC E field perpendicular to its revolution axis: $\mathbf{E}_{\mathbf{0}}=-\mathrm{E}_{0} \mathbf{e}_{\mathbf{x}}$ (figure 2). As described in the introduction, the rotation of the cylinder is explained by the action of the electric field on the charges which have accumulated at its surface. The electric field exerts on the charges that are supposed to be stuck on the cylinder an electric torque (per cylinder unit length) that is written as

$$
\Gamma^{\mathbf{E}}=\mathrm{a}^{2} \int_{0}^{2 \pi} \gamma_{\mathrm{e}}(\theta) \mathrm{E}_{1 \theta}(\rho=\mathrm{a}, \theta) \mathrm{d} \theta \mathbf{e}_{\mathbf{z}}
$$

where $\gamma_{\mathrm{e}}$ is the surface charge distribution.

A conservation equation for the surface charge can be obtained performing a charge balance on an elementary surface, $\mathrm{dS}=\mathrm{a} d \mathrm{~d} \theta$ of the cylinder. The time variation of the charge contained on $\mathrm{dS}$ is given by the sum of two terms:

-The difference of the conduction currents outside and inside the cylinder, crossing dS: $\left(\sigma_{2} \mathbf{E}_{2}-\sigma_{1} \mathbf{E}_{1}\right) \cdot \mathbf{e}_{\rho} \mathrm{d} S$

-The current coming from the displacement of the interfacial charges carried by the rotation of the cylinder: $-\frac{\partial}{\partial \theta}\left(\gamma_{\mathrm{e}} \Omega \mathrm{adz}\right) \mathrm{d} \theta$ where $\Omega$ is the angular velocity of the cylinder around $(\mathrm{Oz})$.

We then obtain:

$$
\frac{\partial \gamma_{\mathrm{e}}}{\partial \mathrm{t}}=\left[\sigma_{1} \frac{\partial \Phi_{1}}{\partial \rho}-\sigma_{2} \frac{\partial \Phi_{2}}{\partial \rho}\right]_{\rho=\mathrm{a}}-\Omega \frac{\partial \gamma_{\mathrm{e}}}{\partial \theta}
$$

where $\Phi_{1}$ and $\Phi_{2}$ denote the electrostatic potentials outside and inside the cylinder which can be split up into two parts: the instantaneous potential created by the charges that are present 
on the electrodes surface, $\Phi_{1,2}^{\infty}$ and the electric potential associated with the superficial charge distribution, $\Phi_{1,2}^{\gamma}$.

Assuming that the charge distribution is dipolar, the potentials are written as:

$$
\begin{aligned}
& \Phi_{1}^{\infty}=\left(\rho-\mathrm{A} \frac{\mathrm{a}^{2}}{\rho}\right) \mathrm{E}_{0} \cos \theta \\
& \Phi_{2}^{\infty}=\rho(1-\mathrm{B}) \mathrm{E}_{0} \cos \theta \\
& \Phi_{1}^{\gamma}=\mathrm{C} \frac{\mathrm{a}^{2}}{\rho} \mathrm{E}_{0} \cos \left(\theta-\theta_{0}\right) \\
& \Phi_{2}^{\gamma}=\mathrm{D} \rho \mathrm{E}_{0} \cos \left(\theta-\theta_{0}\right)
\end{aligned}
$$

where $\theta_{0}$ is the direction of the dipole moment created by the charge distribution and $\mathrm{A} B, \mathrm{C}$ and D are constants. All these five quantities are determined from equation (2) and the boundary conditions:

$$
\begin{aligned}
& \Phi_{1}^{\infty}(\rho=\mathrm{a})=\Phi_{2}^{\infty}(\rho=\mathrm{a}) \\
& \Phi_{1}^{\gamma}(\rho=\mathrm{a})=\Phi_{2}^{\gamma}(\rho=\mathrm{a}) \\
& 0=\left[\varepsilon_{2} \frac{\partial \Phi_{2}^{\infty}}{\partial \rho}-\varepsilon_{1} \frac{\partial \Phi_{1}^{\infty}}{\partial \rho}\right]_{\rho=\mathrm{a}} \\
& \gamma_{\mathrm{e}}=\left[\varepsilon_{2} \frac{\partial \Phi_{2}^{\gamma}}{\partial \rho}-\varepsilon_{1} \frac{\partial \Phi_{1}^{\gamma}}{\partial \rho}\right]_{\rho=\mathrm{a}}
\end{aligned}
$$

Finally, in the stationary regime, we obtain:

$$
\begin{aligned}
& \mathrm{A}=\mathrm{B}=\beta^{\infty} \\
& \mathrm{C}=\mathrm{D}=-\frac{\beta^{0}-\beta^{\infty}}{\sqrt{1+\Omega^{2} \tau_{\mathrm{MW}}^{2}}} \\
& \theta_{0}=\arctan \left(\Omega \tau_{\mathrm{MW}}\right) \text { and } \gamma_{\mathrm{e}}=-\left(\varepsilon_{1}+\varepsilon_{2}\right) \mathrm{CE}_{0} \cos \left(\theta-\theta_{0}\right)
\end{aligned}
$$


where $\tau_{\mathrm{MW}}=\frac{\varepsilon_{1}+\varepsilon_{2}}{\sigma_{1}+\sigma_{2}}$ is the Maxwell Wagner time which is the characteristic time for the interfacial charge (or equivalently for the dipole moment) to relax and $\beta^{\infty}=\frac{\varepsilon_{2}-\varepsilon_{1}}{\varepsilon_{1}+\varepsilon_{2}}$ and $\beta^{0}=\frac{\sigma_{2}-\sigma_{1}}{\sigma_{1}+\sigma_{2}}$ are the Clausius-Mossotti factors of the cylinder at high and zero frequency respectively.

In stationary state, the electric torque (eq.1) is balanced by the resistant viscous torque exerted by the fluid on the cylinder:

$\Gamma^{\mathrm{H}}=-\alpha \Omega$

where $\alpha=4 \pi \eta \mathrm{a}^{2}$ is the rotational friction coefficient of the cylinder (per unit length), $\eta$ being the liquid viscosity.

The stationary behaviour of such a Quincke rotor has been widely studied and shows some interesting features: the rotation of the cylinder depends on a threshold value of the electric field intensity, $E_{c}$, and, above this critical value, the particle rotates around itself with an axis pointing in any direction perpendicular to the field :

$$
\Omega=\frac{1}{\tau_{\mathrm{MW}}} \sqrt{\left(\frac{\mathrm{E}_{0}}{\mathrm{E}_{\mathrm{c}}}\right)^{2}-1} \text { with } \mathrm{E}_{\mathrm{c}}=\sqrt{\frac{-2 \eta}{\tau_{\mathrm{MW}} \varepsilon_{1}\left(\beta^{0}-\beta^{\infty}\right)}}
$$

Furthermore, as mentioned in the introduction, this model predicts that the angular velocity of the cylinder does not depend on its diameter. In the following, we will show that this model is valid only if the thickness of the charge distribution around the cylinder is thin enough compared to the cylinder diameter and if the ion diffusion and electro-migration can be neglected on the cylinder size scale.

\section{Microscopic analysis}




\section{III.A Balance equations}

Modelling Quincke rotation where electric field and fluid dynamic effects are coupled is based on the combination of continuum equations for the electric and the flow fields in the bulk with balance equations for the ion density [13].

The bulk charge distribution around the cylinder and the fluid velocity field are determined upon solving the charge conservation equations and the Navier-Stokes equation in the liquid. Some simplifying assumptions are made: the conductivity of the cylinder is considered to be much smaller than that of the liquid so that $\sigma_{2} \approx 0$ and the dielectric constants of the cylinder and of the liquid are assumed to be equal: $\varepsilon_{1}=\varepsilon_{2}=\varepsilon$. Furthermore, we will restrict our study to low polar materials for which there exist a dynamical equilibrium between free ions and ionic pairs :

$$
\left(\mathrm{A}^{+} \mathrm{B}^{-}\right) \stackrel{\mathrm{k}_{\mathrm{r}}}{\mathrm{k}_{\mathrm{d}}} \mathrm{A}^{+}+\mathrm{B}^{-}
$$

Where $\mathrm{k}_{\mathrm{r}}$ and $\mathrm{k}_{\mathrm{d}}$ are respectively the recombination and the dissociation constants which, at thermodynamic equilibrium, are related by:

$$
\mathrm{k}_{\mathrm{d}} \mathrm{c}_{0}=\mathrm{k}_{\mathrm{r}} \mathrm{n}_{0}^{2}
$$

where $\mathrm{c}_{0}=\left[\mathrm{A}^{+} \mathrm{B}^{-}\right]$and $\mathrm{n}_{0}=\mathrm{n}_{+}=\mathrm{n}_{-}=\left[\mathrm{A}^{+}\right]=\left[\mathrm{B}^{-}\right]$, at thermodynamic equilibrium.

Following Debye [14],

$$
\mathrm{k}_{\mathrm{r}}=\frac{\mathrm{e}_{0}\left(\mathrm{~K}_{+}+\mathrm{K}_{-}\right)}{\varepsilon}\left(1-\exp \left(-\mathrm{l}_{\mathrm{B}} / \mathrm{a}_{\mathrm{i}}\right)\right)^{-1} \text { where } \mathrm{e}_{0} \text { is the elementary charge, } \mathrm{l}_{\mathrm{B}}=\frac{\mathrm{e}_{0}^{2}}{8 \pi \varepsilon \mathrm{kT}} \text {, the }
$$

Bjerrum length and $\mathrm{a}_{\mathrm{i}}$, the distance between ions in an ionic pair ( $\mathrm{k}$ being the Boltzmann constant and $\mathrm{T}$, the temperature). $\mathrm{K}_{+}$and $\mathrm{K}$. are the electrophoretic mobilities of the positive and negative ions which, in the following, are assumed to be the same: $\mathrm{K}_{+}=\mathrm{K}=\mathrm{K}$.

In non-polar fluids, most of the ions are associated in ionic pair $\left(c_{0}>>n_{0}\right)$ and $l_{B}>>a_{i}$ so that the recombination constant reduces to the Langevin constant for gases [15]: 
$\mathrm{k}_{\mathrm{r}} \approx 2 \mathrm{Ke}_{0} / \varepsilon$.

The ion conservation equation for the positive and the negative species is written as [13]:

$\frac{\partial \mathrm{n}_{ \pm}}{\partial \mathrm{t}}+\nabla\left[\mathrm{n}_{ \pm}(\mathbf{v} \mp \mathrm{K} \nabla \Phi)-\mathrm{D} \nabla \mathrm{n}_{ \pm}\right]=\mathrm{k}_{\mathrm{d}} \mathrm{c}-\mathrm{k}_{\mathrm{r}} \mathrm{n}_{+} \mathrm{n}_{-}$

In this equation, $\mathbf{v}$ is the velocity of the fluid and $\mathrm{D}$ is the ionic diffusion coefficient which has been chosen equal for both the positive and the negative ions. $\mathrm{c}$ is the concentration of the neutral species. In the following, it will be considered that, everywhere, the ion concentration, $\mathrm{c}$, is equal to $\mathrm{c}_{0}$ and also to the initial concentration of electrolyte added to the liquid, since, as usual in low polar liquids, the dissociation coefficient is supposed to be small.

The charge density and the electric potential must satisfy Poisson's equation:

$$
\nabla \cdot(\varepsilon \nabla \Phi)=-\mathrm{e}_{0}\left(\mathrm{n}_{+}-\mathrm{n}_{-}\right)
$$

At last, the fluid flow is governed by the Navier-Stokes equation and the continuity equation for an incompressible fluid:

$$
\begin{aligned}
& \rho_{\mathrm{f}} \frac{\partial \mathbf{v}}{\partial \mathrm{t}}+\rho_{\mathrm{f}}(\mathbf{v} . \nabla) \mathbf{v}=-\nabla \mathrm{p}+\eta \nabla^{2} \mathbf{v}-\mathrm{e}_{0}\left(\mathrm{n}_{+}-\mathrm{n}_{-}\right) \nabla \Phi \\
& \nabla . \mathbf{v}=0
\end{aligned}
$$

where $\mathrm{p}$ is the pressure and $\rho_{\mathrm{f}}$, the fluid density.

In a non dimensional form, equations (11), (12), (13) and (14) become:

$$
\begin{aligned}
& \Omega \tau_{\mathrm{r}} \frac{\partial \tilde{\mathrm{n}}_{ \pm}}{\partial \tilde{\mathrm{t}}}+\Omega \tau_{\mathrm{r}} \tilde{\nabla} \cdot\left(\tilde{\mathrm{n}}_{ \pm} \tilde{\mathbf{v}}\right) \mp \frac{\tau_{\mathrm{r}}}{\tau_{\mathrm{m}}} \tilde{\nabla} \cdot\left(\tilde{\mathrm{n}}_{ \pm} \tilde{\nabla} \tilde{\Phi}\right)-\frac{\tau_{\mathrm{r}}}{\tau_{\mathrm{D}}} \tilde{\nabla}^{2} \tilde{\mathrm{n}}_{ \pm}=1-\tilde{\mathrm{n}}_{+} \tilde{\mathrm{n}}_{-} \\
& \tilde{\nabla}^{2} \tilde{\Phi}=-\frac{\tau_{\mathrm{m}}}{2 \tau_{\mathrm{r}}}\left(\tilde{\mathrm{n}}_{+}-\tilde{\mathrm{n}}_{-}\right) \\
& \operatorname{Re}\left(\frac{\partial \tilde{\mathbf{v}}}{\partial \tilde{\mathrm{t}}}+(\tilde{\mathbf{v}} \cdot \tilde{\nabla}) \tilde{\mathbf{v}}\right)=-\tilde{\nabla} \tilde{\mathrm{p}}+\tilde{\nabla}{ }^{2} \tilde{\mathbf{v}}-\frac{\mathrm{r} \tau_{\mathrm{m}}}{2 \Omega \tau_{\mathrm{r}}^{2}}\left(\tilde{\mathrm{n}}_{+}-\tilde{\mathrm{n}}-\right) \tilde{\nabla} \tilde{\Phi} \\
& \tilde{\nabla} \cdot \tilde{\mathbf{v}}=0
\end{aligned}
$$


where the dimensionless variables are:

$\tilde{\mathrm{t}}=\Omega \mathrm{t} ; \quad \tilde{\boldsymbol{\rho}}=\frac{\boldsymbol{\rho}}{\mathrm{a}} ; \quad \tilde{\mathbf{v}}=\frac{\mathbf{v}}{\mathrm{a} \Omega} ; \quad \tilde{\mathrm{n}}_{ \pm}=\frac{\mathrm{n}_{ \pm}}{\mathrm{n}_{0}} ; \tilde{\Phi}=\frac{\Phi}{\mathrm{aE}_{0}}$ and $\tilde{\mathrm{p}}=\frac{\mathrm{p}}{\eta \Omega} . \quad R e=\frac{\rho_{\mathrm{f}} \Omega^{2}}{\eta}$ is the Reynolds number, $r=\left(\frac{E_{0}}{E_{c}}\right)^{2}$ where $E_{c}$ is the threshold field defined in eq.(7). Beside $1 / \Omega, 3$ time scales appear in equations (15)-(17) . $\tau_{\mathrm{r}}=\frac{\varepsilon}{\sigma_{1}}=\frac{\tau_{\mathrm{MW}}}{2}$ is the charge relaxation time, $\tau_{\mathrm{D}}=\frac{\mathrm{a}^{2}}{\mathrm{D}}$ is the characteristic time for the tangential diffusion around the cylinder. At last, $\tau_{\mathrm{m}}=\frac{\mathrm{a}}{\mathrm{KE}_{0}}$ is the characteristic time for ion electro-migration around the cylinder.

Considering eqs. (15)-(17), it is worth noting that $1 / \tau_{\mathrm{m}}$ and $1 / \tau_{\mathrm{D}}$ represent the magnitude of the electro-migration flux and of the tangential diffusion flux respectively.

\section{III.B orders of magnitude}

Now we shall see how the above characteristic times compare to each other for different cylinder sizes. Besides the cylinder size, various physical parameters of the liquid appear in these characteristic times. In the following, we will fix them to the typical values they have in the systems we use to study experimentally [6], [7]: $\varepsilon \approx 2 \varepsilon_{0}, \sigma_{1} \approx 10^{-8} \mathrm{~S} / \mathrm{m}, \eta \approx 10^{-2} \mathrm{~Pa} . \mathrm{s}, \mathrm{K} \approx 10^{-}$ ${ }^{9} \mathrm{~m}^{2} \mathrm{~V}^{-1} \mathrm{~s}^{-1}$. and $\mathrm{D} \approx 2.510^{-11} \mathrm{~m}^{2} \mathrm{~s}^{-1}$. Using these values we get the intensity of the critical field, $\mathrm{Ec}=5.710^{5} \mathrm{~V} / \mathrm{m}$ and the orders of magnitude of the characteristic times which are reported in table 1 for different cylinder radii. It should be noted that whatever the size of the cylinder (comprised between 1 and $100 \mu \mathrm{m}$ ), the diffusion time is always larger than the other ones. But, when the radius of the cylinder becomes as small as $1 \mu \mathrm{m}$, the electro-migration time and the charge relaxation time are of the same order of magnitude and the diffusion time is only 40 times larger than them. So we expect diffusion to play a role for particles smaller than a few microns and electro-migration for particles smaller than a few tens of microns. 


\section{III.C Numerical study}

In this section, we will show how the relative magnitude of the different characteristic times influences the cylinder angular velocity.

\section{Numerical technique.}

The equations have been solved with the software Comsol Multiphysics ${ }^{\mathrm{TM}}$ for an infinite cylinder. The solutions are supposed to be invariant under translation along the cylinder axis (2D approximation). Using the non-dimensional variables of eq. (15)-(18), the cylinder radius equals 1 , and the velocity of the fluid at the surface is purely tangential and equals 1 too. The cylinder is placed at the centre of a square box with edge length 40 . The boundary conditions in the non-dimensional variables are displayed in figure $3 . \mathrm{J}_{\mathrm{n}+}$ and $\mathrm{J}_{\mathrm{n}-\text { are }}$ ar the components of the flux density of ions normal to the boundary. We note that when the characteristic times are varied, the geometry and the boundary conditions are kept unchanged, while the equations are modified.

The mesh is automatically generated by the software. The number of nodes on the cylinder boundary (circle) is fixed, so as to impose the typical size on an element at this position. We usually work with 4000 nodes on the cylinder boundary. As already mentioned, the smaller length of the problem is the charge layer thickness. With 4000 nodes on the boundary, the typical size of an element is $1.510^{-3}$, so that, as it will be shown in section IV, the dense layer is always larger than 3 or 4 times this length. Furthermore, we have checked that the mesh density was sufficient to ensure that the result did not depend on the mesh size.

The non linear solver performs iterative computation, until the relative error estimate was lower than $10^{-3}$, but, usually, the final error estimate is lower than $10^{-6}$.

\section{Numerical procedure}


For each set of parameters $\left\{\left(\frac{E_{0}}{E_{c}}\right)^{2}, \frac{\tau_{m}}{\tau_{r}}, \frac{\tau_{D}}{\tau_{r}}\right\}$, the angular velocity $\Omega$ is increased from zero, and for each value of $\Omega$, the solution of equations $15-18$ is sought and the viscous torque exerted on the cylinder is computed from the fluid velocity field. If the electric field is large enough (higher than a critical value), the viscous torque is positive for low values of $\Omega$, which means that the fluid drives the cylinder (figure 4). For increasing values of $\Omega$, the viscous torque cancels and then becomes negative. The spontaneous rotation velocity is the value of $\Omega$ for which the viscous torque cancels.

\section{Numerical results}

As already mentioned, the purpose of the present paper is to evaluate the effect of the rotor size on its angular velocity. Nevertheless, as it can be seen eq. (15)-(17), the control parameters of the system are the field intensity and the ratios of the electro-migration time and of the diffusion time to the charge relaxation time. As a consequence, in a first step, we shall present an analysis of the electro-mechanical behaviour of the cylinder in terms of the characteristic times. After that, we shall focus on the variation of the cylinder spin rate with its diameter, all other parameters being fixed.

First, we examine the variation of the cylinder spin rate as a function of the squared $\mathrm{E}$ field intensity for various values of the migration time and of the diffusion time (figure 5). One can observe a strong dependence of the angular velocity on both the migration time, $\tau_{\mathrm{m}}$ and the diffusion time, $\tau_{\mathrm{D}}$. The dependence is particularly significant when these two characteristic times are not very large compared to the relaxation time. When it is not the case (i.e. when $\tau_{\mathrm{D}}>>\tau_{\mathrm{r}}$ and $\tau_{\mathrm{m}}>>\tau_{\mathrm{r}}$ ), as expected, the rotor behaviour is very close from that predicted by the Quincke rotation model. At constant $\tau_{\mathrm{D}}$, when the migration time decreases, the rotor spin rate is reduced. The same observation can be made when the diffusion time is 
decreased at constant $\tau_{\mathrm{m}}$ (and when $\tau_{\mathrm{D}}$ is not much larger than $\tau_{\mathrm{m}}$ ). The qualitative interpretation to these two observations is straightforward since the tangential diffusion is expected to smooth the angular dependence of the charge density profile and since the ion electro-migration around the cylinder will decrease the local charge density. Both effects lead to a decrease of the torque exerted by the E field on the ion distribution.

Since the purpose of this paper is to estimate the influence of the rotor size on the Quincke rotation, let us fix all other parameters: $\eta=10^{-2} \mathrm{~Pa} . \mathrm{s}, \varepsilon_{1}=\varepsilon_{2}=2 \varepsilon_{0}, \sigma_{1}=10^{-8} \mathrm{~S} / \mathrm{m}, \mathrm{K}=10^{-}$ ${ }^{9} \mathrm{~m}^{2} \mathrm{~V}^{-1} \mathrm{~s}^{-1}$ and $\mathrm{D}=2.510^{-11} \mathrm{~m}^{2} \mathrm{~s}^{-1}\left(\mathrm{E}_{\mathrm{c}} \approx 570 \mathrm{~V} / \mathrm{mm}\right.$ and $\left.\tau_{\mathrm{r}} \approx 1.8 \mathrm{~ms}\right)$ and let us vary the cylinder radius. The dependence of the cylinder squared spin rate as a function of the normalised squared electric field intensity for different cylinder sizes $(a=2.5,3,5$ and $10 \mu \mathrm{m})$ is shown by the symbols (the meaning of the lines will be given in the next section) in figure 6 . In this representation, the control parameters which are kept constant along each curve of the figure 6 are $\tau_{\mathrm{D}}$ and the product $\mathrm{r} \tau_{\mathrm{m}}^{2}$.

An interesting feature to note on this figure is that, whatever the size of the rotor, the qualitative dependence of the angular velocity on the field intensity is the same as in the classical Quincke model:

$\Omega=\frac{1}{\tau_{\mathrm{MW}}^{*}} \sqrt{\left(\frac{\mathrm{E}_{0}}{\mathrm{E}_{\mathrm{c}}^{*}}\right)^{2}-1}$

where $E_{c}^{*}$ and $\tau_{M W}^{*}$ are function of the parameters $r \tau_{m}^{2}$ and $\tau_{\mathrm{D}}$ or equivalently of the cylinder radius but do not depend on the electric field intensity. The smaller the cylinder radius is, the lower its angular velocity: the critical field increases and the slope of the curves decreases when the size decreases.

Besides this qualitative description, it should be stressed that, in the parameter range we have considered, the tangential diffusion time is always much higher than both the charge 
relaxation and electro-migration times (for $\mathrm{a}=2.5 \mu \mathrm{m}, \tau_{\mathrm{D}} / \tau_{\mathrm{r}}=140$ and $57<\tau_{\mathrm{D}} / \tau_{\mathrm{m}}<228$ for $\mathrm{E}_{\mathrm{c}}<\mathrm{E}_{0}<4 \mathrm{E}_{\mathrm{c}}$; for $\mathrm{a}=10 \mu \mathrm{m}, \tau_{\mathrm{D}} / \tau_{\mathrm{r}}=2200$ and $\left.228<\tau_{\mathrm{D}} / \tau_{\mathrm{m}}<912\right)$. Therefore, consistently with figure 5, the tangential diffusion is not expected to play a significant role. On the opposite, $\tau_{\mathrm{m}}$ is of the same order of magnitude as $\tau_{\mathrm{r}}\left(0.4<\tau_{\mathrm{m}} / \tau_{\mathrm{r}}<1.7\right.$ for $\mathrm{a}=2.5 \mu \mathrm{m}$ and $1.7<\tau_{\mathrm{m}} / \tau_{\mathrm{r}}<6.9$ for $\mathrm{a}=10 \mu \mathrm{m})$. As a consequence, one can guess that the rotor angular velocity is mainly controlled by the electro-migration. In the following section we show how to account for the tangential electro-migration flux in the prediction of the cylinder angular velocity.

\section{Discussion}

In this section, we propose a simple analytical model which holds in the case of negligible tangential diffusion. We show that the migration flux results in an equivalent surface conductivity which can be included in the classical Quincke model. Let us suppose that the surface charge is distributed in a thin layer around the cylinder, so that the ion density is much higher in the layer than in the bulk liquid.

The charge carriers present in this layer are responsible for a local increase of the conductivity. We take it into account by integrating the tangential migration flux over the layer thickness, $\delta$ :

$$
\mathrm{J}_{\mathrm{S}}=\int_{\mathrm{a}}^{\mathrm{a}+\delta}\left(\mathrm{n}_{+}+\mathrm{n}_{-}\right) \mathrm{eKE} \mathrm{E}_{\theta} \mathrm{d} \rho
$$

This expression is further simplified upon supposing that the tangential electric field is constant within the layer and that, whatever the angular position, one type of charge carrier is predominant so that $\mathrm{n}_{+}+\mathrm{n}_{-} \approx\left|\mathrm{n}_{+}-\mathrm{n}_{-}\right|$.

The migration flux is given by:

$$
\mathrm{J}_{\mathrm{S}}=\mathrm{E}_{\theta} \int_{\mathrm{a}}^{\mathrm{a}+\delta}\left|\mathrm{n}_{+}-\mathrm{n}_{-}\right| \mathrm{eKd} \rho \approx\left|\gamma_{\mathrm{e}}(\theta)\right| \mathrm{KE}_{\theta}
$$

where the second approximate equality assumes that the layer is thin $(\delta<<a)$. 
Equation (20) defines a surface conductivity, $\sigma_{\Sigma}(\theta)=\left|\gamma_{\mathrm{e}}(\theta)\right| \mathrm{K}$. For the sake of simplicity, we introduce the average surface conductivity

$$
\sigma_{\Sigma}=\sqrt{\left\langle\gamma_{\mathrm{e}}^{2}\right\rangle_{\theta}} \mathrm{K}
$$

Taking into account this surface conductivity and assuming as in section III that the cylinder is perfectly insulating, equation (2) becomes:

$$
\frac{\partial \gamma_{\mathrm{e}}}{\partial \mathrm{t}}=\left[\sigma_{1} \frac{\partial \Phi_{1}}{\partial \rho}\right]_{\rho=\mathrm{a}}-\Omega \frac{\partial \gamma_{\mathrm{e}}}{\partial \theta}+\frac{\sigma_{\Sigma}}{\mathrm{a}^{2}}\left[\frac{\partial^{2} \Phi_{1}}{\partial \theta^{2}}\right]_{\rho=\mathrm{a}}
$$

Looking for a dipolar solution $\Phi_{2} \square \rho \cos \left(\theta-\theta_{0}\right)$, and using the continuity of the electrostatic potential at the cylinder surface, it follows:

$$
\frac{1}{\mathrm{a}} \frac{\partial^{2} \Phi_{1}}{\partial \theta^{2}}=-\left[\frac{\partial \Phi_{2}}{\partial \rho}\right]_{\rho=\mathrm{a}}
$$

so that equation (22) becomes:

$$
\frac{\partial \gamma_{\mathrm{e}}}{\partial \mathrm{t}}=\left[\sigma_{1} \frac{\partial \Phi_{1}}{\partial \rho}-\frac{\sigma_{\Sigma}}{\mathrm{a}} \frac{\partial \Phi_{2}}{\partial \rho}\right]_{\rho=\mathrm{a}}-\Omega \frac{\partial \gamma_{\mathrm{e}}}{\partial \theta}
$$

Comparing equations (2) and (24), we note that the surface conductivity is equivalent to a bulk conductivity in the cylinder $\sigma_{2}=\sigma_{\Sigma} / \mathrm{a}$ [16]. Supposing $\varepsilon_{1}=\varepsilon_{2}$ as in section III, Equation (5) and (7) together with equations (21) and (24) result in a self-consistent determination of $\sigma_{2}$ :

$$
\begin{aligned}
& \frac{\sigma_{2}}{\sigma_{1}}=\mathrm{R}(\Omega)=\sqrt{2} \frac{\tau_{\mathrm{r}}}{\tau_{\mathrm{m}}} \frac{1-\mathrm{R}}{1+\mathrm{R}} \frac{1}{\sqrt{1+\left(\frac{\Omega \tau_{\mathrm{MW}}}{1+\mathrm{R}}\right)^{2}}} \\
& \left(\frac{\Omega \tau_{\mathrm{MW}}}{1+\mathrm{R}}\right)^{2}=\mathrm{r} \frac{1-\mathrm{R}}{(1+\mathrm{R})^{2}}-1
\end{aligned}
$$

Eliminating $\Omega$ in the equations (25), the expression of the normalized equivalent bulk conductivity in the cylinder is obtained: 


$$
\frac{\sigma_{2}}{\sigma_{1}}=\frac{1}{r\left(\frac{\tau_{\mathrm{m}}}{\tau_{\mathrm{r}}}\right)^{2}}\left(\sqrt{1+2 \mathrm{r}\left(\frac{\tau_{\mathrm{m}}}{\tau_{\mathrm{r}}}\right)^{2}}-1\right)
$$

This expression deserves one important remark: the equivalent bulk conductivity is a function of the single parameter $\mathrm{r}\left(\tau_{\mathrm{m}} / \tau_{\mathrm{r}}\right)^{2}$. In particular, turning to the physical parameters, $r\left(\frac{\tau_{m}}{\tau_{r}}\right)^{2}=\frac{\sigma_{1}}{4 \eta K^{2}} a^{2}$ so that $\sigma_{2} / \sigma_{1}$ does not depend on the electric field intensity, but on the cylinder radius. Thus, for a given rotor size, the angular velocity obeys equation (7) with the constant cylinder conductivity (26). We note that $\tau_{\mathrm{D}}$ does not appear in eq.(26). Indeed, the tangential diffusion flux has been neglected.

The results of this model together with the numerical computation are displayed on figure 6 . Firstly, concerning the intermediate sizes 10 and 5 microns, corresponding to values of $\mathrm{r}\left(\tau_{\mathrm{m}} / \tau_{\mathrm{r}}\right)^{2}$ equal to 100 and 25 respectively, the surface conduction model agrees quite convincingly with the f.e.m. computation, even as the latter differs sensibly from the Quincke model. However, for high field intensities, we note a slight deviation from the linear behaviour. When $\mathrm{r}\left(\tau_{\mathrm{m}} / \tau_{\mathrm{r}}\right)^{2}$ decreases and becomes smaller than a typical value of 10 , the discrepancies grow up, and the model turns out to be inadequate.

To try to better understand this discrepancy, we can examine the numerical results concerning the radial charge distribution that are displayed in figure 7 for two values of the cylinder radius $(\mathrm{a}=10 \mu \mathrm{m}$ and $\mathrm{a}=2.5 \mu \mathrm{m})$. It appears clearly that, whatever the angular velocity of the cylinder, the ionic cloud is much more extended when the cylinder is small. More precisely, apart from the very dense and thin layer close to the surface, there exists a diffuse layer which can be much thicker. For instance, the normalized charge density has decayed to about one-thousandth of its surface value at a typical normalized distance of 0.15 for $\mathrm{a}=10 \mu \mathrm{m}$ and 1.5 for $\mathrm{a}=2.5 \mu \mathrm{m}$. Furthermore, for this last rotor size, in the direction where the charge 
density is maximum, only approximately half the charge is contained in the dense layer. In that case, the thin layer approximation may be very crude. In particular, the velocity field of the liquid around the particle, which is coupled to the charge distribution, will be quite different from the $1 / \rho$ velocity profile that is supposed to take place in the Quincke rotation model and that results in the viscous torque expression (6). More generally, all the fluxes may play a role in the building of the charge layer. For that reason, it seems difficult to give an analytical description of the system, and even to evaluate the thickness of the layer. Anyway, we have checked that it could be as large as ten times the Debye length $\left(\sqrt{\mathrm{D} \tau_{\mathrm{r}}}=\mathrm{a} \sqrt{\frac{\tau_{\mathrm{r}}}{\tau_{\mathrm{D}}}}\right)$.

However, an order of magnitude of the dense layer thickness, $\delta$, may be obtained by equating the radial diffusion and migration ion fluxes at the insulating cylinder surface:

$$
\begin{aligned}
& \mathrm{Kn}_{ \pm} \mathrm{E}_{0} \sim \mathrm{D} \mathrm{n}_{ \pm} / \delta \\
& \frac{\delta}{\mathrm{a}} \square \frac{\tau_{\mathrm{m}}}{\tau_{\mathrm{D}}}
\end{aligned}
$$

Analysing the numerical computation of the charge profiles, we have been able to confirm that eq. (28) gives the right order of magnitude for the dense layer thickness. Indeed, in table 2, one can see a comparison between the results provided on one hand by the expression (28) and on the other hand by the analysis of the numerical charge profiles in the direction where the charge density is maximum, for different values of $r$ and a. From a numerical point of view, the layer thickness is evaluated by assuming that the charge density profile is exponential and by dividing its value at the cylinder surface by e, the Euler's number.

Again, for cylinder sizes larger than typically five microns, the charge is mainly contained in the thin layer, and the thin layer hypothesis holds. On the opposite, for smaller sizes, both thick and thin layer have to be considered, and the electro-mechanical behaviour of the system has to be studied numerically. 


\section{Conclusion}

Using a finite element method, a numerical study of Quincke rotation has been performed in order to evaluate the influence of the rotor size on its angular velocity when subjected to a DC electric field. To solve this problem, the charge conservation equations and the Navier-Stokes and Poisson equations have been written in a non-dimensional form where, beside the inverse of the cylinder spin rate, three characteristic times appear: the diffusion time and the ion electro-migration time over the rotor radius, and the charge relaxation time. A study of the influence of the magnitude of these different characteristic times has been addressed and it has been shown that both electro-migration and diffusion result in a decrease of the angular velocity of the cylinder and an increase of the threshold field. A modified Quincke model has been proposed to calculate analytically the rotor spin rate. This model accounts for the electro-migration inside the charge layer which is present around the rotor thanks to an equivalent surface conductivity. Its results agree with the numerical ones if the charge layer thickness is small compared to the cylinder radius. The numerical computation shows that it is the case if the cylinder radius is larger than typically five microns. For smaller particles, the estimation of the charge thickness may be difficult due to the large number of coupled parameters that play a role (electro-migration, diffusion, convection). However, we have shown that the charge layer consists in a dense and thin layer at the cylinder surface surrounded by a diffuse layer whose thickness can be as large as ten times the Debye length..

\section{Acknowledgments}

The authors would like to thank Alberto T. Perez and Pierre Atten for the interest they paid on this work and for very helpful discussions. 


\section{References}

[1] G. Quincke, "Ueber rotationen im constanten electrischen felde", Ann. Phys. Chem., 59, 417-486 (1896)

[2] P.E. Secker and I.N. Scialom, J. Appl. Phys., 39, pp. 2957-61, (1968).

[3] J.R. Melcher, IEEE Trans.Educ., E-17, pp. 100-110, (1974).

[4] T.B. Jones, IEEE Trans. IAS IA-20, pp.845-849, (1984).

[5] T. Coddington, A. F. Pollard and H. House, "Operation of a dielectric motor with a low conductivity liquid", J. Phys. D: Appl. Phys., 4, 1212-1218 (1970)

[6] N. Pannacci, E. Lemaire and L. Lobry, "How insulating particles increase the conductivity of a suspension", Phys. Rev. letters, 99, 094503.1-4 (2007a).

[7] E. Lemaire, L. Lobry, N. Pannacci and F. Peters, J. Rheol., 52, pp.769-783 (2008).

[8] R. H. Leach, R. J. Pierce, E. P. Hickman, M. J. Mackenzie and H. G. Smith, "The Printing Ink Manual", chap. 12, Ed. Springer Netherlands (2007).

[9] C. F. Wilson, M. I. Wallace, K. Morishima, G. J. Simpson and R. N. Zare, "Coupled Electrorotation of Polymer Microspheres for Microfluidic Sensing and Mixing", Anal. Chem., 74, 5099-5104 (2002).

[10] Huang J.P., Yu K.W., Gu G.Q., "Electrorotation of colloidal suspensions, Phys. Lett. A 300, 385-391 (2002).

[11] Zhou H., Preston M. A. Tilton R. D., White L. R., "Calculation of the electric polarizability of a charged spherical dielectric particle by the theory of colloidal electrokinetics", J. Colloid Interface Sci., 285, 845-856 (2005).

[12] Zimmerman V., Shilov V. N., Lopez-Garcia J. J. and Grosse C., "Numerical calculation of the electrorotation velocity of latex-type paricles", J. Phys. Chem., 13384-392 (2002). 
[13] Castellanos A, Zhakin A.I.., Watson P.K., Atten P. and J.S. Chang, "Electrohydrodynamics", part II, Ed. Springer-Verlag Wien New York (1998) .

[14] Debye P., "Reaction rates in ionic solutions", Trans. Electrochem. Soc., 82, 262-272 (1942)

[15] Langevin P., "Recombinaison et mobilité des ions dans les gaz", Ann. Chim. Phys., 28, 433 (1903).

[16] Jones T.B., "Electromechanics of particles" Cambridge University Press (1995) 


\section{Table caption}

Table 1. Variation of the 3 characteristic times with the radius of the rotor. The electric field intensity is $10^{6} \mathrm{~V} / \mathrm{m}$ and the physical parameters used to calculate these times are: $\varepsilon_{1}=\varepsilon_{2} \approx 2 \varepsilon_{0}$, $\sigma_{1} \approx 10^{-8} \mathrm{~S} / \mathrm{m}, \eta \approx 10^{-2} \mathrm{~Pa} . \mathrm{s}, \mathrm{K} \approx 10^{-9} \mathrm{~m}^{2} \mathrm{~V}^{-1} \mathrm{~s}^{-1}$. and $\mathrm{D} \approx 2.510^{-11} \mathrm{~m}^{2} \mathrm{~s}^{-1}$.

Table 2. Thin layer thickness around the rotating cylinder for various cylinder radii and various field intensities: a comparison between the layer thickness obtained thanks to the numerical computation $(\delta / a)$ and the order of magnitude resulting from the balance of the radial diffusion and the tangential electro-migration fluxes $\left(\tau_{\mathrm{m}} / \tau_{\mathrm{D}}\right)$. 


\section{Figure caption}

Figure1. If the charge relaxation time is larger in the particle than in the liquid, the superficial charge distribution is equivalent to that of a dipole in the direction opposite to the DC E field (a). The particle starts rotating to flip its dipole in the field direction. In stationary regime, the dipole forms a constant non zero angle with the field (b).

Figure 2. An insulating cylinder (symmetry axis along $\mathrm{z}$ ) is submitted to a DC electric field pointing in the (-x) direction.

Figure 3. Boundary conditions for the normalized equations. An insulating cylinder (normalized radius, 1) is placed in a square box (normalized edge length, 40).

Figure 4. Normalized viscous torque of the cylinder versus its angular velocity. This curve has been obtained for $\left(\mathrm{E}_{0} / \mathrm{E}_{\mathrm{c}}\right)^{2}=4, \tau_{\mathrm{m}} / \tau_{\mathrm{r}}=2.5$ and $\tau_{\mathrm{D}} / \tau_{\mathrm{r}}=564$.

Figure 5. Squared normalized spin rate versus $r=\left(E_{0} / E_{C}\right)^{2}$ for different values of $\tau_{m} / \tau_{r}$ and $\tau_{\mathrm{D}} / \tau_{\mathrm{r}}:$ solid lines: $\tau_{\mathrm{D}} / \tau_{\mathrm{r}}=2000$, dashed lines: $\tau_{\mathrm{D}} / \tau_{\mathrm{r}}=200$, dotted lines: $\tau_{\mathrm{D}} / \tau_{\mathrm{r}}=20$, circles: $\tau_{\mathrm{m}} / \tau_{\mathrm{r}}=20$, triangles: $\tau_{\mathrm{m}} / \tau_{\mathrm{r}}=2$,squares: $\tau_{\mathrm{m}} / \tau_{\mathrm{r}}=0.8$. The thick line corresponds to the predictions of the classical Quincke rotation model.

Figure 6. Squared normalized spin rate versus $r=\left(E_{0} / E_{C}\right)^{2}$ for different values of the cylinder radius. The lines correspond to the predictions of the model and the symbols to the numerical computations: circles, dashed line: $\mathrm{a}=10 \mu \mathrm{m}\left(\mathrm{r}\left(\tau_{\mathrm{m}} / \tau_{\mathrm{r}}\right)^{2}=100\right)$; triangles, dotted line: $\mathrm{a}=5 \mu \mathrm{m}$ $\left(\mathrm{r}\left(\tau_{\mathrm{m}} / \tau_{\mathrm{r}}\right)^{2}=25\right)$; squares, dot-dashed line: $\mathrm{a}=3 \mu \mathrm{m}\left(\mathrm{r}\left(\tau_{\mathrm{m}} / \tau_{\mathrm{r}}\right)^{2}=11\right)$; stars, solid line: $\mathrm{a}=2.5 \mu \mathrm{m}$ 
$\left(\mathrm{r}\left(\tau_{\mathrm{m}} / \tau_{\mathrm{r}}\right)^{2}=6.25\right)$. The thick line corresponds to the predictions of the classical Quincke rotation model.

Figure 7. Normalized charge density profile for two values of the cylinder radius. The diffuse layer normalized thickness is far larger in the case of the smallest cylinder. 


\begin{tabular}{|c|c|c|c|c|}
\hline Cylinder radius $(\mu \mathrm{m})$ & 1 & 2.5 & 10 & 100 \\
\hline$\tau_{\mathrm{r}}=\frac{\varepsilon}{\sigma}(\mathrm{s})$ & $1.810^{-3}$ & $1.810^{-3}$ & $1.810^{-3}$ & $1.810^{-3}$ \\
\hline$\tau_{\mathrm{m}}=\frac{a}{K E_{0}}(\mathrm{~s})$ & $10^{-3}$ & $2.510^{-3}$ & $10^{-2}$ & $10^{-1}$ \\
\hline$\tau_{\mathrm{D}}=\frac{a^{2}}{D}(\mathrm{~s})$ & $410^{-2}$ & 0.25 & 4 & 400 \\
\hline
\end{tabular}

Table 1 


\begin{tabular}{|l|c|c|c|c|r|}
\hline & $\mathrm{r}=2$ & $\mathrm{r}=4$ & $\mathrm{r}=9$ & $\mathrm{r}=16$ & \\
\hline \multirow{2}{*}{$\mathrm{a}=10 \mu \mathrm{m}$} & $2.410^{-3}$ & $2.810^{-3}$ & $3.610^{-3}$ & & $\delta / \mathrm{a}$ \\
\cline { 2 - 6 } & $3.210^{-3}$ & $2.210^{-3}$ & $1.510^{-3}$ & & $\tau_{\mathrm{m}} / \tau_{\mathrm{d}}$ \\
\hline \multirow{2}{*}{$\mathrm{a}=5 \mu \mathrm{m}$} & $4.810^{-3}$ & $410^{-3}$ & $410^{-3}$ & & $\delta / \mathrm{a}$ \\
\cline { 2 - 6 } & $6.310-3$ & $4.410^{-3}$ & $2.910^{-3}$ & & $\tau_{\mathrm{m}} / \tau_{\mathrm{d}}$ \\
\hline \multirow{2}{*}{$\mathrm{a}=3 \mu \mathrm{m}$} & & $5.610^{-3}$ & $4.410^{-3}$ & $3.210^{-3}$ & $\delta / \mathrm{a}$ \\
\cline { 2 - 6 } & & $7.410^{-3}$ & $4.910^{-3}$ & $3.710^{-3}$ & $\tau_{\mathrm{m}} / \tau_{\mathrm{d}}$ \\
\hline \multirow{2}{*}{$\mathrm{a}=2.5 \mu \mathrm{m}$} & & & $5.210^{-3}$ & $4.410^{-3}$ & $\delta / \mathrm{a}$ \\
\cline { 2 - 6 } & & & $5.910^{-3}$ & $4.410^{-3}$ & $\tau_{\mathrm{m}} / \tau_{\mathrm{d}}$ \\
\hline
\end{tabular}

Table 2 


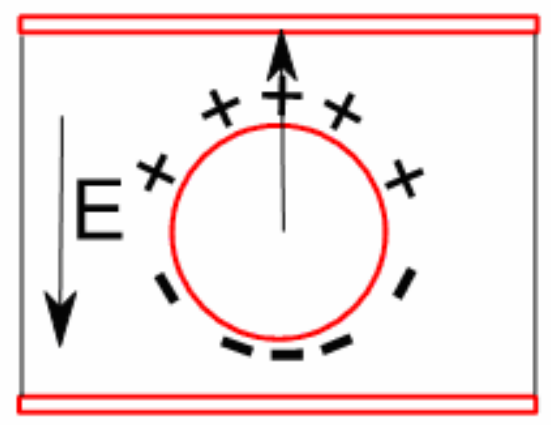

Figure 1.a

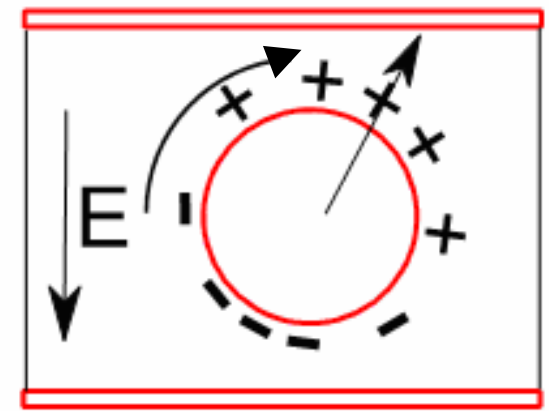

Figure 1.b 


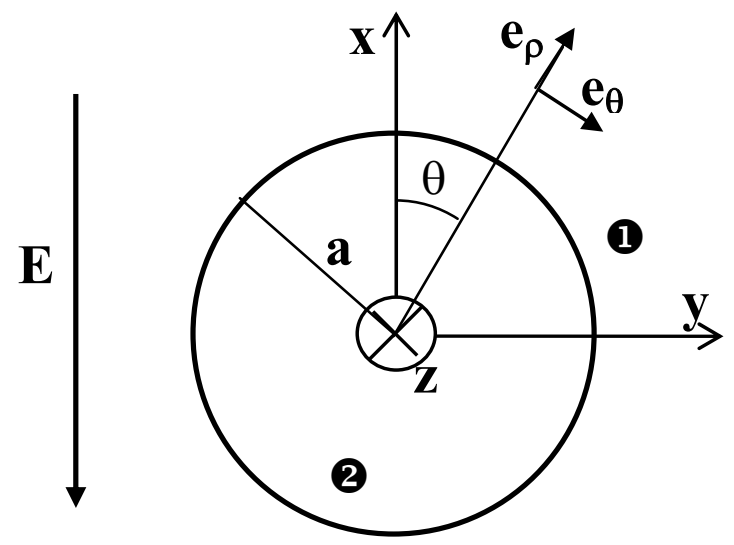

Figure 2 


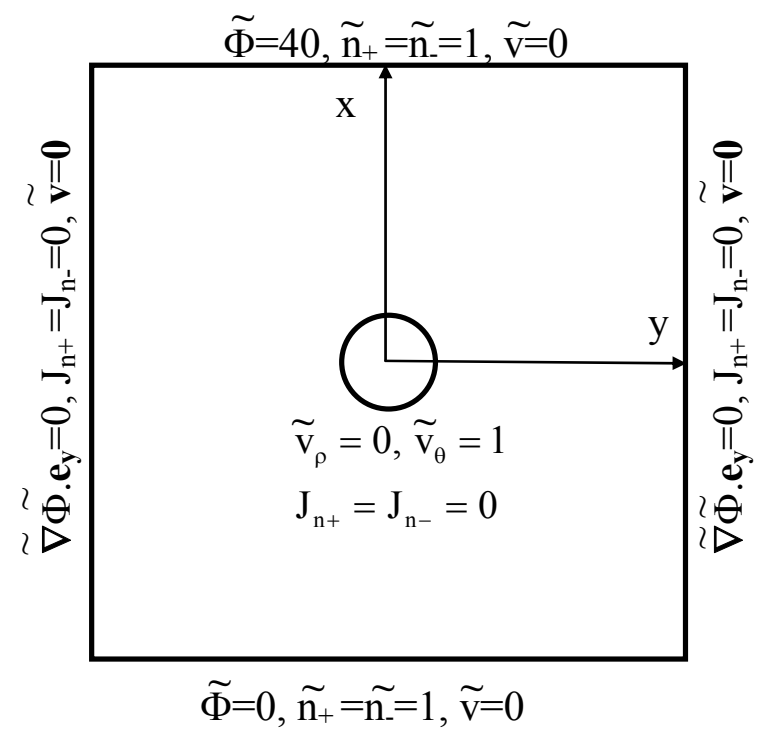

Figure 3 


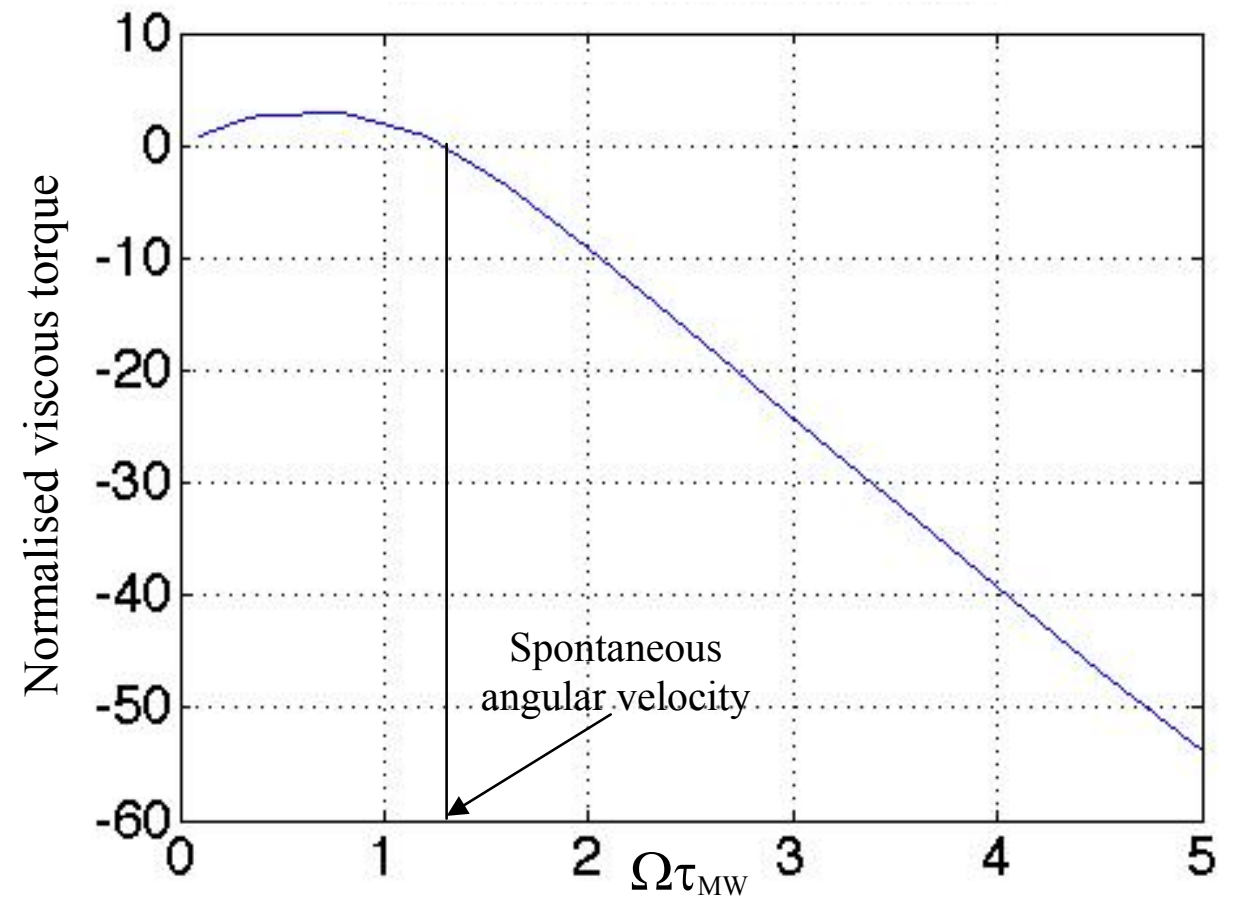

Figure 4 


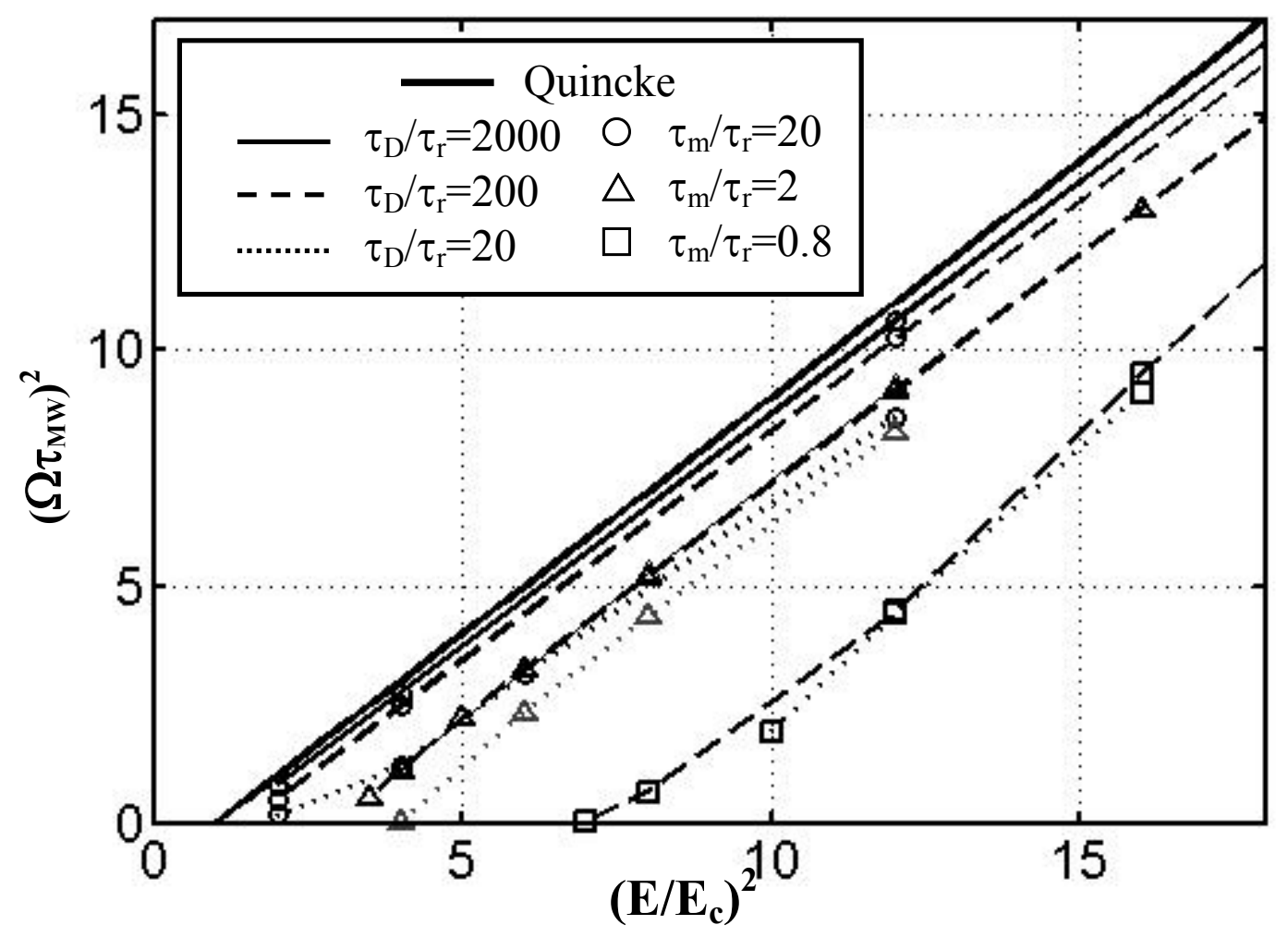

Figure 5 


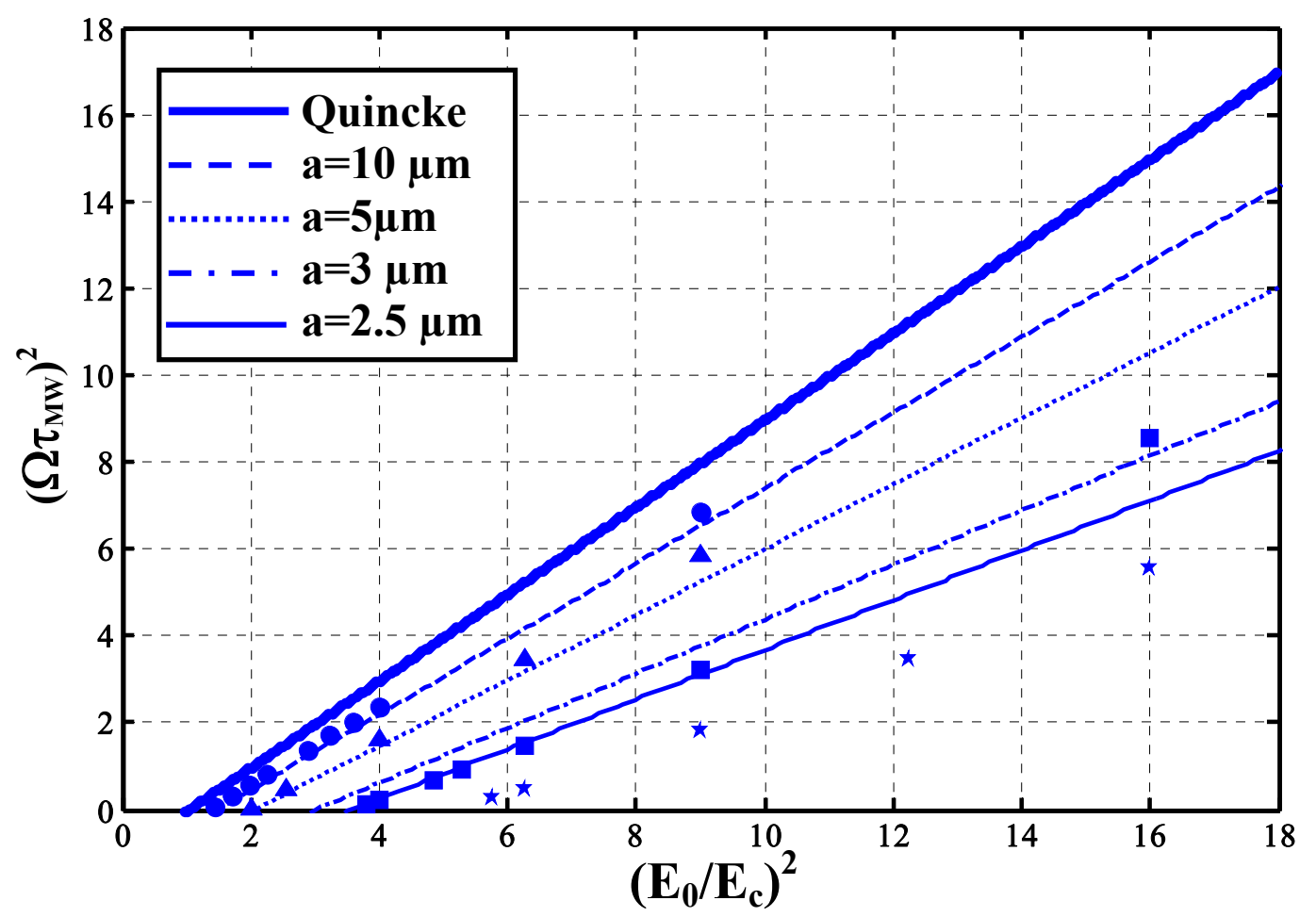

Figure 6 


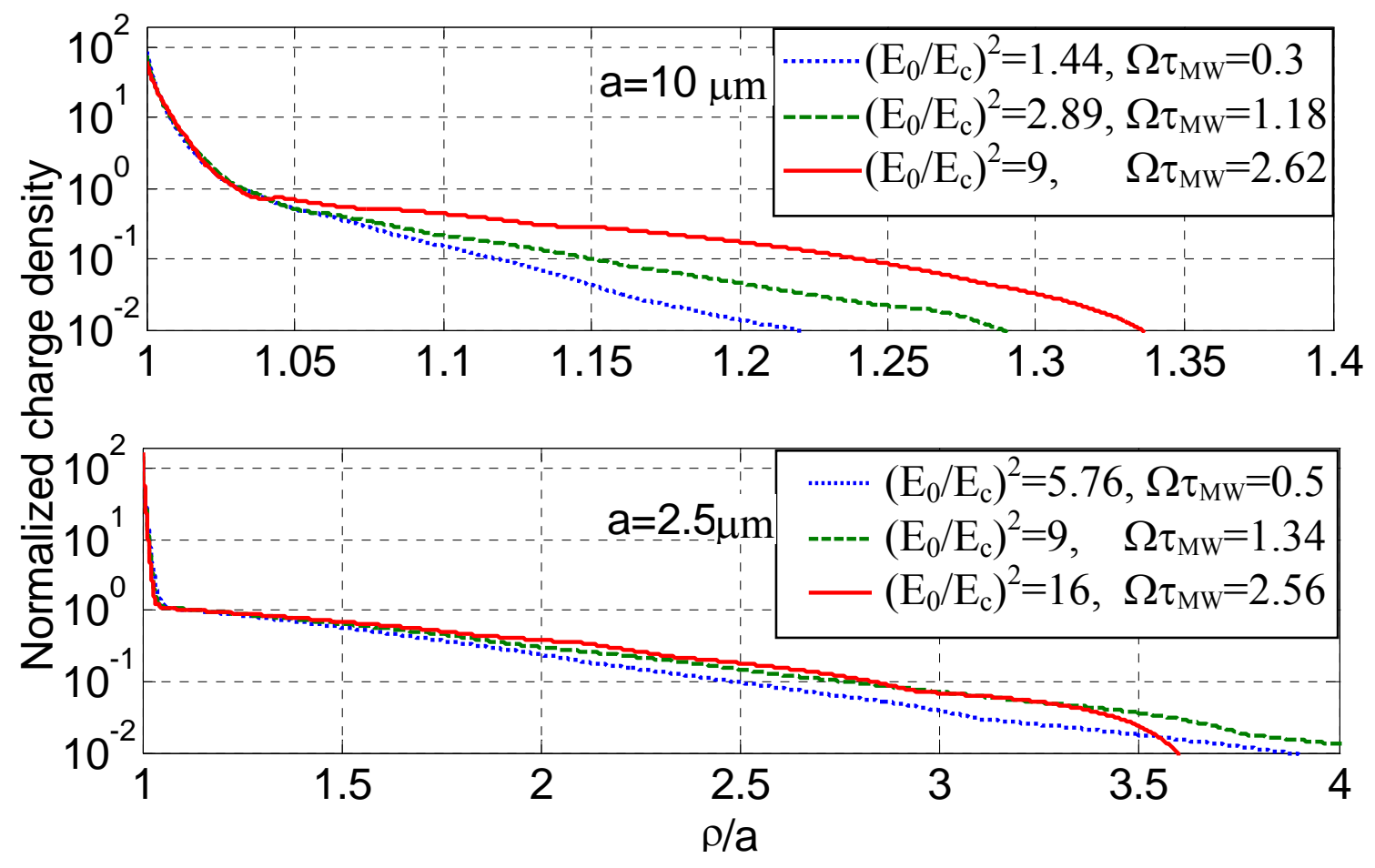

Figure 7 